\title{
What is the thalamus in zebrafish?
}

\author{
Thomas Mueller * \\ Department of Developmental Biology, Faculty of Biology, Institute of Biology I, University of Freiburg, Freiburg, Germany
}

\section{Edited by:}

Steffen Scholpp, Karlsruhe Institute of Technology, Germany

\section{Reviewed by:}

Sylvie Retaux, Centre National de la Recherche Scientifique, France

Mario F. Wullimann,

Ludwig-Maximilians-Univerisät

Munich, Germany

\section{*Correspondence:}

Thomas Mueller, Department of Developmental Biology, Faculty of Biology, Institute of Biology I, University of Freiburg, Hauptstrasse 1, D-79104 Freiburg, Germany. e-mail: thomas.mueller@biologie. uni-freiburg.de

Current research on the thalamus and related structures in the zebrafish diencephalon identifies an increasing number of both neurological structures and ontogenetic processes as evolutionary conserved between teleosts and mammals. The patterning processes, for example, which during the embryonic development of zebrafish form the thalamus proper appear largely conserved. Yet also striking differences between zebrafish and other vertebrates have been observed, particularly when we look at mature and histologically differentiated brains. A case in point is the migrated preglomerular complex of zebrafish which evolved only within the lineage of ray-finned fish and has no counterpart in mammals or tetrapod vertebrates. Based on its function as a sensory relay station with projections to pallial zones, the preglomerular complex has been compared to specific thalamic nuclei in mammals. However, no thalamic projections to the zebrafish dorsal pallium, which corresponds topologically to the mammalian isocortex, have been identified. Merely one teleostean thalamic nucleus proper, the auditory nucleus, projects to a part of the dorsal telencephalon, the pallial amygdala. Studies on patterning mechanisms identify a rostral and caudal domain in the embryonic thalamus proper. In both, teleosts and mammals, the rostral domain gives rise to GABAergic neurons, whereas glutamatergic neurons originate in the caudal domain of the zebrafish thalamus. The distribution of GABAergic derivatives in the adult zebrafish brain, furthermore, revealed previously overlooked thalamic nuclei and redefined already established ones. These findings require some reconsideration regarding the topological origin of these adult structures. In what follows, I discuss how evolutionary conserved and newly acquired features of the developing and adult zebrafish thalamus can be compared to the mammalian situation.

Keywords: isocortex, forebrain, ray-finned fish, reticular thalamic nucleus, teleost, thalamic eminence, thalamocortical

\section{INTRODUCTION}

The thalamus of mammals and other vertebrates is a prominent, multinucleated structure in the diencephalon (Jones, 2007;

\footnotetext{
Abbreviations: A, anterior thalamic nucleus; $\mathrm{AO}$, anterior octaval nucleus; aP1, alar plate prosomere 1; aP2, alar plate prosomere 2; aP3, alar plate prosomere 3; BLA, basolateral amygdala; BNSM, bed nucleus of the stria medullaris; bP1, basal plate prosomere 1; bP2, basal plate prosomere 2; $\mathrm{bP} 3$, basal plate prosomere 3; chor, commissura horizontalis; $\mathrm{CN}$, cochlear nucleus; $\mathrm{CP}$, caudate putamen; $\mathrm{CP}^{\circ}$, centroposterior thalamic nucleus; cpost, commissura posterior; cTh, caudal thalamus proper; Ctx, isocortex; D, dorsal telencephalon (pallium); Dc, central zone of the dorsal telencephalon; Dl, lateral zone of the dorsal telencephalon; DON, descending octaval nucleus; dot, dorsomedial optic tract; Dm, medial zone of the dorsal telencephalon; DP, dorsal pallium; Dp, posterior zone of the dorsal telencephalon; $\mathrm{DP}^{\circ}$, dorsoposterior thalamic nucleus; Th, thalamus (proper); E, epiphysis; EmT, eminentia thalami; EN, entopeduncular nucleus; fr, fasciculus retroflexus; GP, globus pallidus; $\mathrm{H}$, hypothalamus; $\mathrm{Ha}$, habenula; Had, dorsal habenular nucleus; Hav, ventral habenular nucleus; Hd, dorsal zone of the periventriuclar hypothalamus; Hip, hippocampus; $\mathrm{Hv}$, ventral zone of periventricular nucleus; I, intermediate thalamic nucleus; IC, intercalated thalamic nucleus; InCo, inferior colliculus; lfb, lateral forebrain bundle; LH, lateral hypothalamic nucleus; lot, lateral olfactory tract; LP, lateral pallium; $\mathrm{LP}^{\mathrm{o}}$, lateroposterior thalamic complex; $\mathrm{MGN}$, medial geniculate nucleus; $\mathrm{MO}$, medullar oblongata; $\mathrm{MP}$, medial pallium; $\mathrm{OB}$, olfactory bulb; oc, optic commissure; $\mathrm{P}$, pallium; $\mathrm{P}^{\mathrm{o}}$, posterior thalamic (preglomerular) nucleus; $\mathrm{P} 1$, prosomere 1; P2, prosomere 2; P2, prosomere 3; PG, preglomerular complex; PGa, anterior preglomerular nucleus; PGc, caudal preglomerular nucleus; PGl, lateral preglomerular nucleus; PGm, medial preglomerular nucleus; pirCtx, piriform cortex; Po, preoptic region; $\mathrm{PPa}$, parvocellular preopticnucleus, anterior part; $\mathrm{PPv}$,
}

Nieuwenhuys et al., 2007). Often called the "gateway to consciousness," the thalamus regulates attention and alertness. As an interface between isocortex and deeper brain structures, the thalamus distributes, modifies, and filters ascending and descending information from and to various parts of the brain. Due to its relevance in human brain pathology, the thalamus is best studied in mammalian systems, particularly in rodents (hamster, mouse, rat, cats, and primates; Jones, 2007). According to these studies, the mammalian thalamus is functionally subdivided into four types of nuclei: sensory relay, motor, associative, and limbic ones. All

periventricular pretectal nucleus, ventral part; $\mathrm{PPd}$, periventricular pretectal nucleus, dorsal part; PPp, parvocellular preoptic nucleus, posterior part; Pr, pretectum; PSm, magnocellular superficial pretectal nucleus; PSp, parvocellular superficial pretectal nucleus; PT, posterior tuberculum; PTd, dorsal posterior tubercular region; PVO, paraventricular organ; rTh, rostral thalamus proper; RTN, reticular thalamic nucleus; S, subpallium; SC, suprachiasmatic nucleus; SD, saccus dorsalis; SG, subglomerular nucleus; SO, superior olive; SOP, secondary octaval population; SuCo, superior colliculus; Teg, tegmentum; TeO, tectum opticum; TGN, tertiary gustatory nucleus; TLa, torus lateralis; TPB, thalamo-pallial border; TPp, periventricular nucleus of posterior tuberculum; TPm, migrated nucleus of posterior tuberculum; TS, torus semicircularis; TSc, central nucleus of torus semicircularis; $\mathrm{V}$, ventral telencephalon (subpallium); VAO, ventral accessory optic nucleus; $\mathrm{Vd}$, dorsal nucleus of the area ventralis; VL, ventrolateral thalamic nucleus; VM, ventromedial thalamic nucleus; VP, ventral pallium; VT, ventral thalamus; Y, sulcus ypsiloniformis; ZLI, zona limitans intrathalamica. 
of these nuclei hold neurons that project to the isocortex. In fact, the thalamus and the isocortex in placental mammals are tightly correlated as two important findings highlight: firstly, lesions of particular cortical areas or removal of the whole isocortex lead to the degeneration of thalamic nuclei (Rose and Woolsey, 1943; Loopuijt et al., 1995; Kaas, 2009). Secondly, mammals with a less differentiated and smaller isocortex exhibit a less nucleated and prominent thalamus (Jones, 2007).

Hence, the thalamus and its connections to the isocortex are considered crucial to the evolution of human cognition and behavior. Comparative neurobiologists are trying to reconstruct the evolutionary history of the thalamus through cladistic outgroup comparisons and the identification of shared characters in diverse non-tetrapod anamniotes. Evolutionary developmental (evo-devo) studies, in contrast, focus on anamniote model organisms by comparing gene expression patterns and molecular compositions that define primordial brain structures. Among these model organisms, the teleost zebrafish has gained particular importance because of the availability of a large number of mutants and transgenic lines. In comparison to zebrafish, basally derived actinopterygians such as bichirs and sturgeons (Figure 1) are developmentally much less investigated. Frequently, the absence of detailed data about outgroups of teleosts undermines the determination of a character in question as being homologous or convergent to that of another group of species. However, differences in molecular composition and developmental history can be strong arguments against certain homologies (e.g., the teleostean anterior thalamic nucleus versus the mammalian dorsal lateral geniculate nucleus, (dLGN; see below).

This review compares the zebrafish thalamus (Figure 2) with the one of mammals. And while my focus will be on the thalamus proper, I also discuss surrounding thalamic regions and thalamus-like structures and their connections to the pallium. I do so because fundamental differences between the zebrafish and the mammalian forebrain make a direct comparison both impossible and inadequate. For example, defining features of the mammalian thalamus proper, such as the thalamocortical connections, are inexistent in teleosts. In fact, teleosts lack a sophisticated

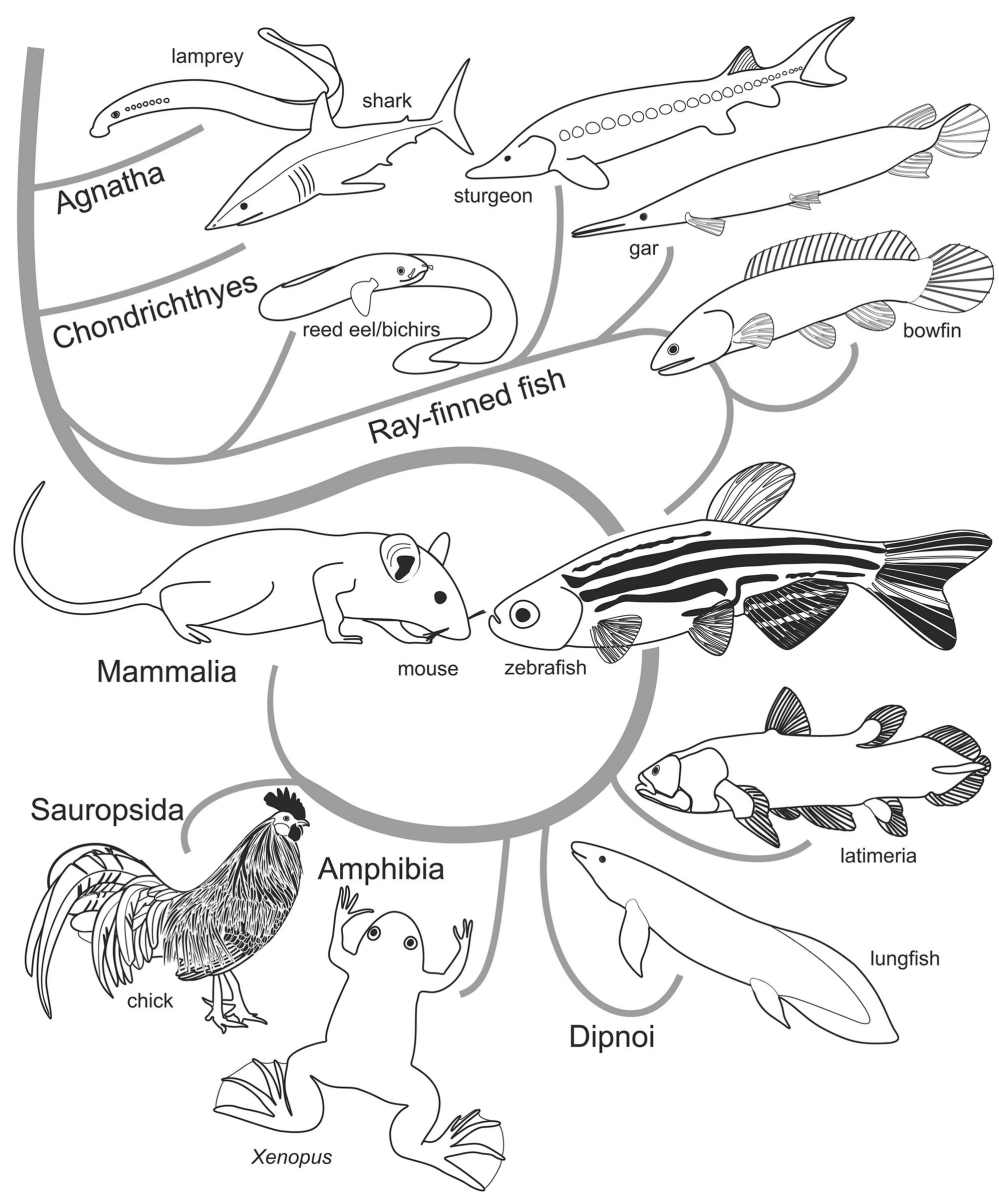

FIGURE 1 | Cladogram showing relationships of extant vertebrates.

Determining homologies in brains of distantly related species, such as zebrafish (teleosts) and mouse (mammals), relies on comparative knowledge in regard to the ancestral situation provided by outgroup comparisons (Hennig, 1966). That is, to establish a neural character in zebrafish as homologous to a topologically corresponding structure in the mammalian brain, the neural character needs to be present in the last common (extinct) ancestor of these species. A high likelihood for a structure being homologous is given when the character in question exists in basally emerged ray-finned fish such as bichirs, sturgeons, and gars and in anamniote tetrapods (amphibians) and sauropsids (birds and reptiles). 


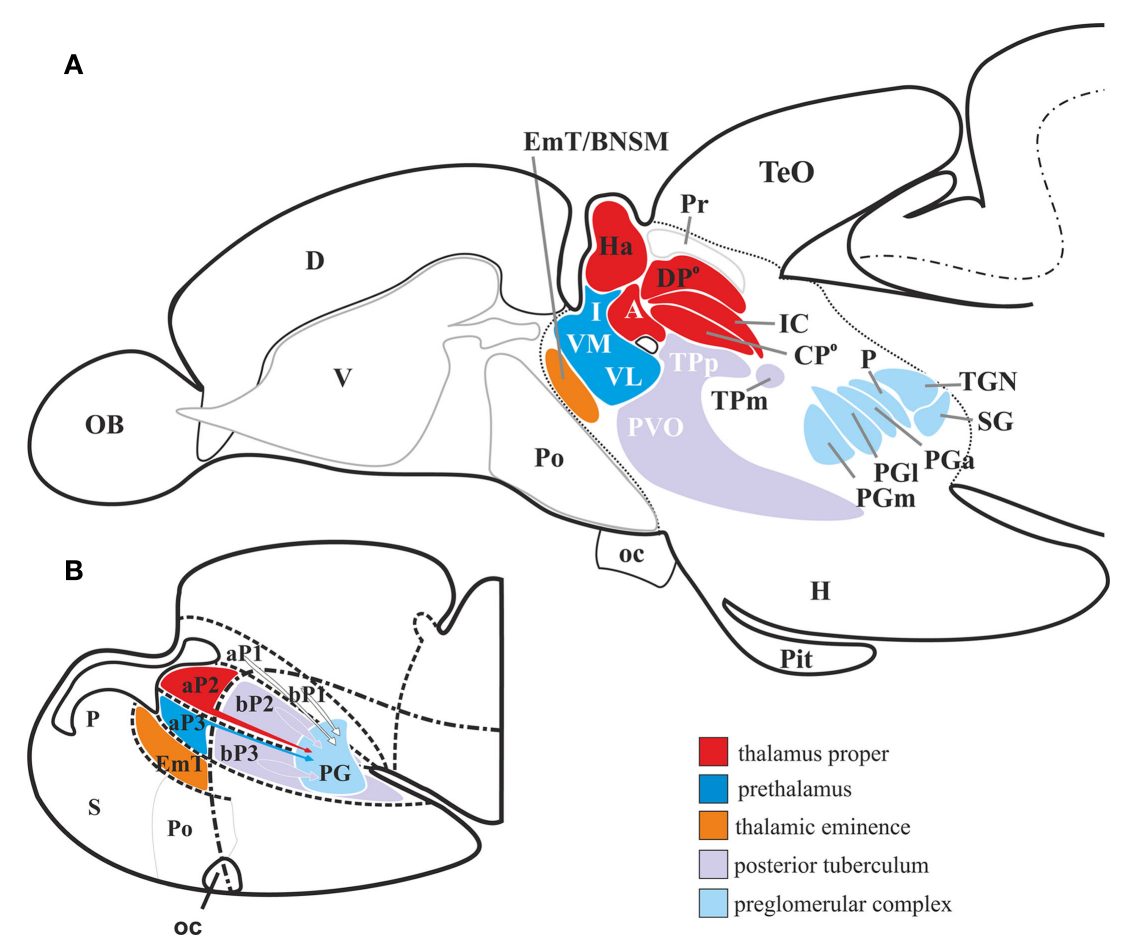

FIGURE 2 | Schematic drawing of the wider thalamus in the larval and adult zebrafish. (A,B) The alar plate prosomere 2 [aP2, red in (B)] derived dorsal thalamic nuclei [red in (A)] are located dorsally to the zona limitans intrathalamica. These are the habenular nuclei, the auditory dorsoposterior $\left(\mathrm{DP}^{\circ}\right)$, the intercalated $(\mathrm{IC})$, the visual centroposterior $\left(\mathrm{CP}^{\circ}\right)$, and the anterior thalamic nuclei (A). The alar plate prosomere 3 (aP3) derived ventral thalamic nuclei (blue) are the intermediate (I), the ventromedial (VM), and the ventrolateral $(\mathrm{VL})$ thalamic nuclei. The posterior tubercular nuclei are derived from basal plate portions of prosomere 2 and $3(b B 2+3)$. The nuclei of the preglomerular complex [light blue in (A)], which together serve as the major thalamo-like sensory relay station, are likely of multiprosomeric origin [indicated with arrows in (B)]. Abbreviations see list. six-layered isocortex, which in mammals provides chief instances of sensory processing. Teleosts, however, do possess a dorsal pallial division that topologically corresponds to the mammalian isocortex (Figure 3). Yet, the zebrafish dorsal pallium does not hold sensory areas that receive projections from relay stations comparable to the mammalian thalamus proper (Mueller et al., 2011). For example, the auditory thalamic nucleus $\left(\mathrm{CP}^{\mathrm{o}}\right)$ of teleosts projects to the amygdala (Dm) and to the hippocampal (Dl) division, regions that are, like their mammalian counterparts, involved in emotional response behaviors and spatial orientation respectively (Portavella et al., 2002; Northcutt, 2006). The thalamus proper is also less prominent in zebrafish than in mammals. This is the case because teleosts possess a preglomerular complex, an elaborated migrated agglomeration of nuclei related to the posterior tuberculum absent in mammals. In fact, the preglomerular complex serves as a predominant sensory relay station in the teleostean diencephalon (Wullimann and Northcutt, 1990; Northcutt, 2008; Yamamoto and Ito, 2008).

I discuss in what follows the thalamus proper and structures of the caudal diencephalon that form what I call the wider thalamus. My approach aims at relating similarities and differences in the zebrafish brain to defining features of the mammalian thalamus proper. First, I examine the prosomeric Bauplan of the zebrafish forebrain and recent findings that subdivide the thalamus proper into a rostral and a caudal part. Then I look at brain regions that qualify either as thalamus proper or as thalamus-like structures.
My closing focus is placed on the functional organization of two ascending sensory systems in teleosts: the acoustic and the visual. While the auditory pathway gives a case of conservation, the visual pathway illustrates a case of non-conservation.

\section{OVERVIEW}

In this review, I consider the forebrain of carp-like (cyprinid) teleosts such as zebrafish and goldfish as representative for their fish clade. The cyprinid forebrain is relatively simple in structure, which facilitates the comparison with other vertebrates (Rupp et al., 1996). What is more, most of the developmental data on the teleostean forebrain comes from zebrafish research. In contrast, most of the connectional and functional data stem from studies of goldfish. I look at developmental and hodological data of zebrafish and goldfish to arrive at a generalized picture of the cyprinid forebrain. While a forebrain comparison across the teleostean clade exceeds the focus of my contribution, excellent reviews have already addressed such a comparison (Braford and Northcutt, 1983; Northcutt and Davis, 1983; Northcutt and Wullimann, 1988; Nieuwenhuys and Meek, 1990; Meek and Nieuwenhuys, 1998).

With the term wider thalamus, I refer to the thalamus proper (formerly "dorsal thalamus"), the prethalamus (formerly "ventral thalamus"), the thalamic eminence, the periventricular posterior tuberculum, and the migrated preglomerular complex. This broad definition corresponds to the one used by Bergquist (1932), who 

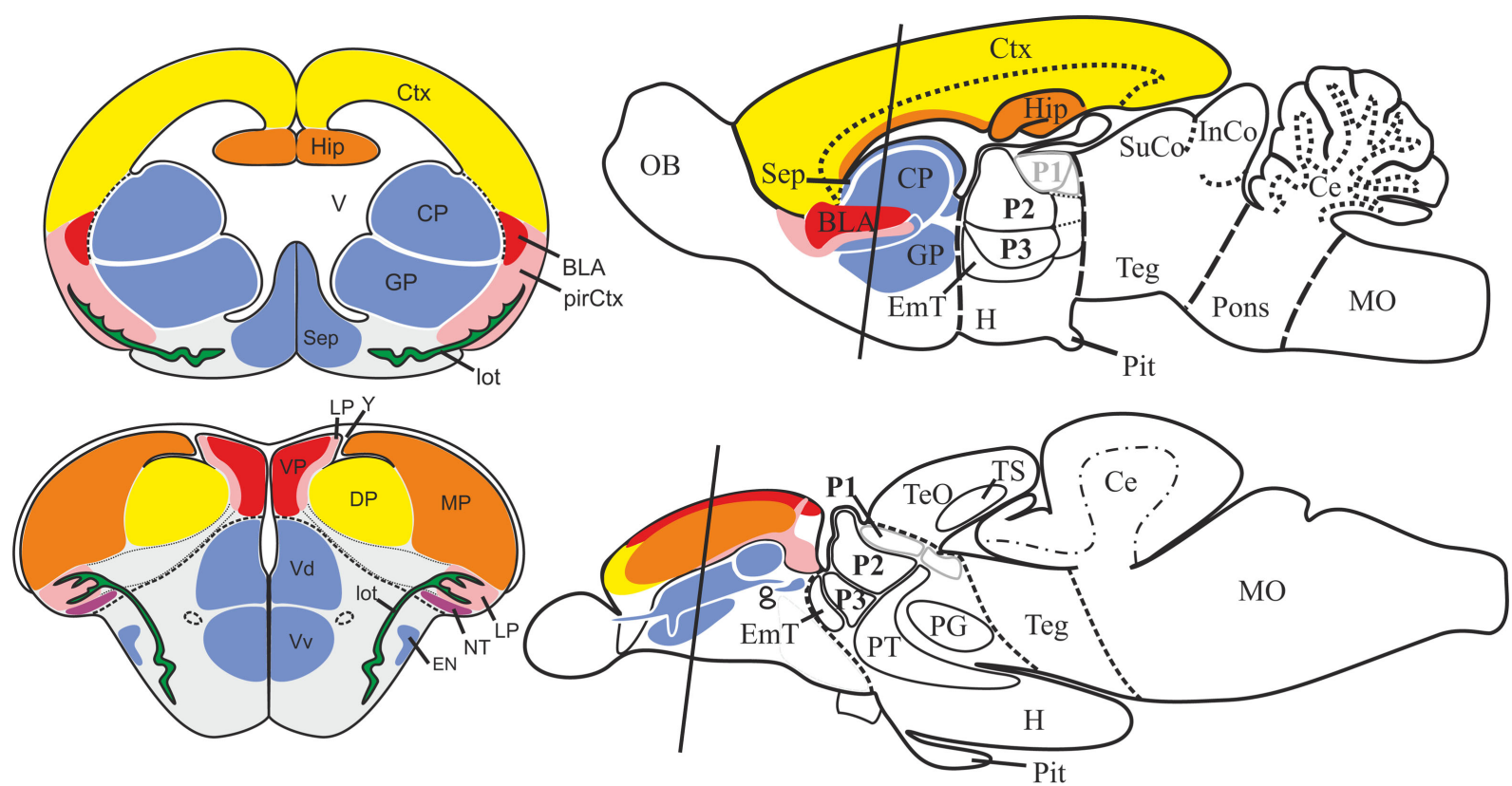

ventral pallium (VP)

lateral pallium (LP)

dorsal pallium (DP)

medial pallium (MP)

subpallium

FIGURE 3 | Schematic drawing illustrating topological correspondences between cyprinid (zebrafish/goldfish; teleostean) and rodent (mouse/rat; mammalian) telencephala. The zebrafish telencephalon is characterized by two massive lobes covered by a dorsally located $T$-shaped ventricle. The mouse telencephalon, in contrast, consists of two bilateral hemispheres surrounding centrally located ventricles. The topology of the zebrafish pallium is similar to the one in mouse and can be explained through topographical shifts of its constituting pallial divisions during a complex outward folding process. The zebrafish pallium, like its mammalian counterpart, consists mainly of four pallial divisions: a medial pallium (MP) homologous to the mammalian hippocampus (Hip), a dorsal pallial division (DP) topologically corresponding to the mammalian isocortex (Ctx), and ventral (VP) and lateral (LP) pallial divisions homologous to the mammalian pallial (basolateral) amygdala (BLA) and piriform cortex (pirCtx) respectively. After Mueller et al. (2011). Abbreviations see list. first described the segmental organization of the longitudinally bent vertebrate forebrain. Bergquist's work is still foundational to the prosomeric forebrain model we use today (Puelles and Rubenstein, 1993, 2003). In the nomenclature of the zebrafish diencephalon I follow Braford and Northcutt (1983) with some modifications by Wullimann and colleagues (Wullimann et al., 1996; Rink and Wullimann, 2004; Wullimann and Mueller, 2004a).

Teleosts clearly share with other vertebrates the thalamus proper, the habenula, and the prethalamus (Figure 2A). According to classical comparative works (Bergquist, 1932; Nieuwenhuys, 1963; Braford and Northcutt, 1983; Wullimann, 1998), the thalamus proper in zebrafish is subdivided into the anterior thalamic nucleus $(\mathrm{A})$, the dorsal posterior thalamic nucleus $\left(\mathrm{DP}^{\mathrm{O}}\right)$, the central posterior thalamic nucleus $\left(\mathrm{CP}^{\circ}\right)$, and the ventral (Hav) and dorsal (Had) habenular nuclei. The ventral and dorsal habenular nuclei are homologous to the mammalian medial and lateral habenular nuclei respectively (Amo et al., 2010). Some prethalamic structures are present in zebrafish but absent in the mammalian brain. These are the intermediate (I), the ventromedial (VM), and the ventrolateral (VL) thalamic nuclei. These nuclei can be found in amphibians as well but not in mammals (Braford and Northcutt, 1983; Neary and Northcutt, 1983; Butler and Northcutt, 1993; Rupp and Northcutt, 1998).
The posterior tuberculum and the preglomerular complex (Figure 2A) are two dominant structures in the basal diencephalon of zebrafish, which also have no apparent counterparts in amniote tetrapods like birds and mammals. Yet, a large posterior tuberculum is present in jawless vertebrates (lampreys), cartilaginous fishes (sharks and manta rays), lungfish, and amphibians (Nieuwenhuys et al., 1998). In mammals, the basal plate derivatives of prosomere two and three are homologous to the dorsal and ventral posterior tubercular fields of developing zebrafish (Wullimann and Puelles, 1999). In the mature zebrafish brain, the posterior tuberculum comprises the periventricular nucleus of the posterior tuberculum with its characteristic large pear-shaped dopaminergic cells, the paraventricular organ, and the posterior tuberal nucleus. All of them are intercalated between prethalamus and hypothalamus (Rupp et al., 1996; Wullimann et al., 1996). Some of these dopaminergic neurons found in the posterior tuberculum of zebrafish project to the subpallium and have been compared and homologized with an anteriormost diencephalic, not mesencephalic, division of the mammalian ascending mesodiencephalic dopaminergic groups A8-A10 in mammals (Wullimann and Rink, 2001; Rink and Wullimann, 2002). Other studies demonstrate that the majority of the dopaminergic neurons in the posterior tuberculum depend on the expression of Orthopedia (Otp) 
and Nkx2.1 homeodomain proteins, similar to A11-dopaminergic groups present in the pretectum and thalamus proper of mammals (Ryu et al., 2006, 2007). These A11-dopaminergic neurons are the major far-projecting dopaminergic neurons that in zebrafish project to the subpallium, the diencephalon, the hindbrain, and the spinal cord similar to the situation in mammals (Tay et al., 2011).

The preglomerular complex (Figure 2A) consists of the anterior, the lateral, the medial, and the caudal preglomerular nuclei (PGa, PGl, PGm, PGc). It also comprises the tertiary gustatory nucleus (TGN), the so-called posterior thalamic (P), and the subglomerular (SG) nucleus. These nuclei have been interpreted as migrated derivatives of the embryonic posterior tuberculum (Braford and Northcutt, 1983; Northcutt, 2008). They serve as sensory relay stations projecting to different parts of the pallium, comparable to sensory thalamic nuclei in mammals. Based on their projection patterns to pallial parts of the adult zebrafish telencephalon and on expression patterns of pax6 in larval zebrafish, these nuclei have been interpreted as homologous to thalamic nuclei of mammals (Yamamoto and Ito, 2008). The diencephalic organization of teleosts differs also gross anatomically from the one of mammals due to the dominance of the periventricular posterior tuberculum and the migrated preglomerular complex.

\section{THE PROSOMERIC MODEL}

During the last decades, a number of evolutionary and developmental (evo-devo) studies have addressed questions of forebrain homologies across vertebrates through the comparison of gene expression patterns. In the developing mouse brain, for example, patterning genes such as Otx1/2, Pax2/6, Emx1/2, Dlx1/2, $N k \times 2.1 / 2.2$, and $s h$, define longitudinal and transverse forebrain domains across all vertebrate clades. Initially, the vertebrate forebrain has been interpreted as being divided into six transverse units called prosomeres (Puelles and Rubenstein, 1993). A modified version with only three prosomeres and non-prosomeric telencephalic and hypothalamic parts serves today as the standard for cross-species comparisons (Wullimann and Puelles, 1999; Puelles and Rubenstein, 2003). This new framework of the prosomeric model subdivides the forebrain into a posterior prosomeric diencephalon (P1-P3) and an anterior non-prosomeric telencephalon and hypothalamus. Figure $\mathbf{2 B}$ shows the schematic division of the prosomeric forebrain of larval zebrafish. The caudal diencephalon, furthermore, consists of alar and basal plate portions. The thalamus proper is a developmental derivative of the alar plate prosomere two (aP2, red in Figure 2B). In longitudinal perspective, the thalamus proper is placed anteriorly to both the pretectum, which originates from the alar plate prosomere one $(\mathrm{aP} 1)$, and the prethalamus, which originates from the alar plate prosomere three (aP3; blue in Figure 2B). Thalamus proper and prethalamus ventrally border with posterior tubercular portions, i.e., basal plate of prosomeres two (bP2) and three (bP3), respectively. In mammals, these basal plate regions develop into the fields of Forel and the retromammillary area (Puelles and Rubenstein, 2003).

In zebrafish and other teleosts, an agglomeration of nuclei, the preglomerular complex, flanks the periventricular posterior tuberculum. In the past, the preglomerular complex (light blue in Figure 2A) has been treated as a basal plate derivative of the posterior tuberculum (Bergquist, 1932). It is, however, more likely that other alar and basal plate territories such as the pretectum (aP1), the nucleus of the medial longitudinal fascicle (bP1), the thalamus ( $\mathrm{aP} 2)$, and the prethalamus ( $\mathrm{aP} 3$ ) contribute cells to the preglomerular complex through radial and tangential migration. Immunohistological stainings against proliferating cell nuclear antigen (PCNA) and bromodeoxyuridine (BrdU) longterm labels on brain sections from larvae between 2 and 5 days postfertilization have shown small chains of migratory cells between different alar and basal plate portions and the primordial preglomerular complex (M2; Wullimann and Puelles, 1999; Mueller and Wullimann, 2002). The expression of pax6 in the developing brain of both zebrafish and medaka also suggest that alar plate 2 and 3 contribute neurons to the preglomerular complex (Wullimann and Rink, 2001; Ishikawa et al., 2007). Yet, the development of the preglomerular complex has not been studied in detail. In fact, the place of origin of none of its nuclei has been determined through fate mapping. This is why I provisionally treat the preglomerular complex as an entity with undetermined place of origin and designate it a migrated (=lateral) neighbor of the (periventricular) posterior tuberculum.

Recent findings regarding the zebrafish thalamus proper require a slight modification of the prosomeric model. In particular, studies on patterning mechanisms demonstrate that the development of the thalamus proper is more complex than previously assumed (Scholpp and Lumsden, 2010). In mammals, diffusible sonic hedgehog $(S h h)$ molecules released from the middiencephalic organizer (MDO) in the zona limitans intrathalamica (ZLI) and FGF signaling released by a dorsal signaling source influence the development of the anterodorsal part of the thalamus (Kataoka and Shimogori, 2008; Scholpp et al., 2009; Vue et al., 2009; Scholpp and Lumsden, 2010). The combinatorial action of these morphogens leads to the production of GABAergic cell groups in the thalamus proper, which otherwise consists of glutamatergic cells. Based on these findings, the thalamus proper is now subdivided in a rostral (rTh) and a caudal (cTh) domain. The rostral part gives rise to the predominantly GABAergic intergeniculate leaflet (IGL) and contributes GABA cell populations to the ventral geniculate nucleus (Vue et al., 2007, 2009). The majority of the glutamatergic relay nuclei, such as the (dLGN; visual), the medial geniculate nucleus (MGN; auditory), and the ventrobasal complex (somatosensory) likely develop from the caudal portion of the thalamus proper (cTh; Jones and Rubenstein, 2004).

The thalamic eminence (EmT), an ambiguous thalamic structure, is located in the optic stalk area at the border of pallium and thalamus (zebrafish: orange in Figures 2A,B). This region is called the telencephalon impar (Nieuwenhuys et al., 2007). In the mammalian brain, the EmT is visible only during developmental stages. In the original prosomeric model, the EmT develops from the alar plate of prosomere four (aP4; Puelles and Rubenstein, 1993). Only in amphibian species like salamanders and frogs is the EmT prominent also in the adult brain. I treat the EmT as a developmental unit lying anterior to the prethalamus in the longitudinal view as proposed in the original prosomeric model (Puelles and Rubenstein, 1993; Wullimann and Puelles, 1999; Mueller and Wullimann, 2009). More recently, Puelles and Rubenstein (2003) have argued that the EmT is a dorsal part of the prethalamus. Since then, a number of studies have placed the EmT within the 
prethalamus. However, studies have shown that prethalamus and EmT can be clearly distinguished based on their distinct molecular profiles during early phases of neurogenesis (Wullimann and Mueller, 2004a; Osório et al., 2010).

\section{THE WIDER THALAMUS IN ADULT ZEBRAFISH}

Molecular mechanisms patterning the thalamus proper appear highly conserved across vertebrates (Scholpp and Lumsden, 2010). Like in its mammalian counterpart, the zebrafish thalamus proper during early developmental stages divides into a rostral (rTh) and a caudal (cTh) domain (Scholpp et al., 2009; Peukert et al., 2011). The rostral zone is defined by expression of ascl1, a basic helixloop-helix transcription factor (bHLH-TF) coding gene involved in the production and patterning of GABAergic phenotypes. The caudal zone (cTh), in contrast, is defined by expression of neurog1, which encodes for a bHLH-TF determining glutamatergic phenotypes. Both neuronal phenotypes depend on different levels of sonic hedgehog (shh) released by the MDO. High concentrations of shh and the presence of her6 promotes GABAergic cell fates whereas low levels and the absence of her6 induces glutamatergic thalamic neurons (Scholpp et al., 2009; Scholpp and Lumsden, 2010). How these early patterns of GABAergic versus glutamatergic territories relate to the adult distribution is still unclear. While only detailed and complex lineage studies could produce here reliable answers, the prosomeric distribution of GABAergic territories across vertebrates allows the proposition of some reasonable hypotheses in regard to the topology of the zebrafish thalamus (Brox et al., 2003; Mueller et al., 2006; Robertson et al., 2007).

In what follows, I describe the zebrafish thalamus proper as a largely glutamatergic derivative of the prosomere 2 (aP2) clearly distinguishable from the GABAergic prethalamus of the prosomere 3 (aP3) lying anteriorly to the ZLI (Figure 4B; Figure 4E for overview). There are, however, some GABAergic nuclei lying posteriorly to the ZLI that may be interpreted as derivatives of the embryonic rostral thalamus proper (rTh/aP2). In contrast, the majority of the nuclei classically treated as thalamus proper likely are predominantly glutamatergic derivatives of the caudal thalamus (cTh/aP2).

Two GABAergic nuclei in the thalamus proper lying close to the ZLI appear to originate in the rostral thalamus proper ( $\mathrm{rTh}$ ): the anterior thalamic nucleus (A) and the only recently recognized intercalated thalamic nucleus (IC; Mueller and Guo, 2009).

The anterior thalamic nucleus (A, Figures 4B,C) of cyprinids and other teleosts topologically corresponds to the anterior nucleus of amphibians. It likely processes emotional and, according to some authors also visual, information (Braford and Northcutt, 1983; Neary and Northcutt, 1983). It still remains to be determined how the anterior thalamic nucleus, a putative derivative of the rostral thalamus proper, relates to mammalian structures derived from this domain. In rodents, the rostral thalamus develops to the IGL and contributes to the ventral geniculate nucleus. The latter, though, is primarily a derivative of the prethalamus (Nakagawa and O'Leary, 2001; Brox et al., 2003; Jones and Rubenstein, 2004; Vue et al., 2007, 2009; Mueller and Guo, 2009; Inamura et al., 2011). In zebrafish neither the ventral lateral geniculate nucleus (vLGN) nor the IGL have been identified. Some authors suggest that the anterior thalamic nucleus of zebrafish is field homologous to the dLGN in mammals, because the anterior thalamic nucleus is located in a topological similar position (Butler's "lemnothalamus"; Butler, 1995; Butler and Hodos, 2005). The mammalian dorsal geniculate nucleus, however, is predominantly glutamatergic and thus probably belongs to the caudal thalamus (cTh). The teleostean GABAergic anterior thalamus is, furthermore, unlikely homologous to nuclei of the mammalian anterior thalamic complex, because the latter also most probably belongs to the cTh although detailed fate mapping studies are still missing (compare Vue et al., 2009).

The previously unrecognized GABAergic intercalated thalamic nucleus (IC; Figure 4D) is sandwiched between dorsoposterior $\left(\mathrm{DP}^{\mathrm{o}}\right)$ and centroposterior $\left(\mathrm{CP}^{\mathrm{o}}\right)$ nuclei. It is possible that the IC develops from the rostral thalamus (rTh) due to its position close to the ZLI. Fate mapping studies still need to confirm this hypothesis. The IC has not been found in any other anamniote vertebrate. Its delineation in zebrafish drastically reduces the extent of the dorsoposterior and centroposterior nuclei and redefines their borders.

Most of the thalamic nuclei are free of GABAergic neurons, as can be expected for glutamatergic derivatives of the cTh. To this group belong the habenular (Hav, Had; Figure 4A), the visual dorsoposterior $\left(\mathrm{DP}^{\mathrm{o}}\right.$; Figures $\left.4 \mathrm{C}, \mathrm{D}\right)$, and the auditory centroposterior $\left(\mathrm{CP}^{\circ}\right.$; Figure $\left.4 \mathrm{D}\right)$ thalamic nuclei.

In adult zebrafish brains derivatives of the prethalamus are GABAergic derivatives which lie anteriorly to the ZLI. They are the ventromedial (VM), the ventrolateral (VL), and the intermediate (I) thalamic nuclei (Figure 4A). These nuclei are present only in anamniotes but not in mammals (Braford and Northcutt, 1983).

Finally, the putative reticular thalamic nucleus (RTN; Figures 4B,C) in zebrafish is a lose GAD67-positive GABAergic aggregation of cells surrounding the anterior $(\mathrm{A})$ and dorsoposterior $\left(\mathrm{DP}^{\mathrm{O}}\right)$ thalamic nuclei (Figures $4 \mathrm{~B}, \mathrm{C}$ ). It is unclear, if this nucleus is homologous to the one in mammals. The mammalian $\mathrm{RTN}$, a derivative of the prethalamus (P3), lies rostroventrally adjacent to the sensory thalamus proper (P2) of adult rodents and other mammals. Topographically, the mature zebrafish RTN sits in a similar position and is closely associated with the sensory thalamus proper. It is possible that the zebrafish RTN exerts similar modulatory functions to the one in mammals. Both the topological origin and the function of the zebrafish RTN, however, are undefined (Mueller and Guo, 2009).

In larval zebrafish, the EmT (Figures 5A-D,F) has been located based on expression patterns of neuroD and eomesA (Wullimann and Mueller, 2004a). In zebrafish and mammals, the EmT appears to give rise to the glutamatergic bed nucleus of the stria medullaris (BNSM; Mueller and Guo, 2009). The BNSM in adult zebrafish sits in the stalk (peduncular) region, a transitional area at the border to the telencephalon impar (Mueller and Guo, 2009). In adult zebrafish the remainder of the EmT has not yet been identified. As Figures 5E1+2 show, the EmT lies within the optic stalk area close to the BNSM. It is a periventricularly located proliferative cell mass located ventrally to the caudalmost telencephalon. Both EmT and BNSM are GAD67-negative (Figures 5E1+2; Figure 5G for overview). What I define as EmT in the mature zebrafish has previously been misinterpreted as a telencephalic proliferation zone. In the atlas of the adult zebrafish brain, for example, the EmT is considered the proliferative zone of the posterior pallial nucleus 

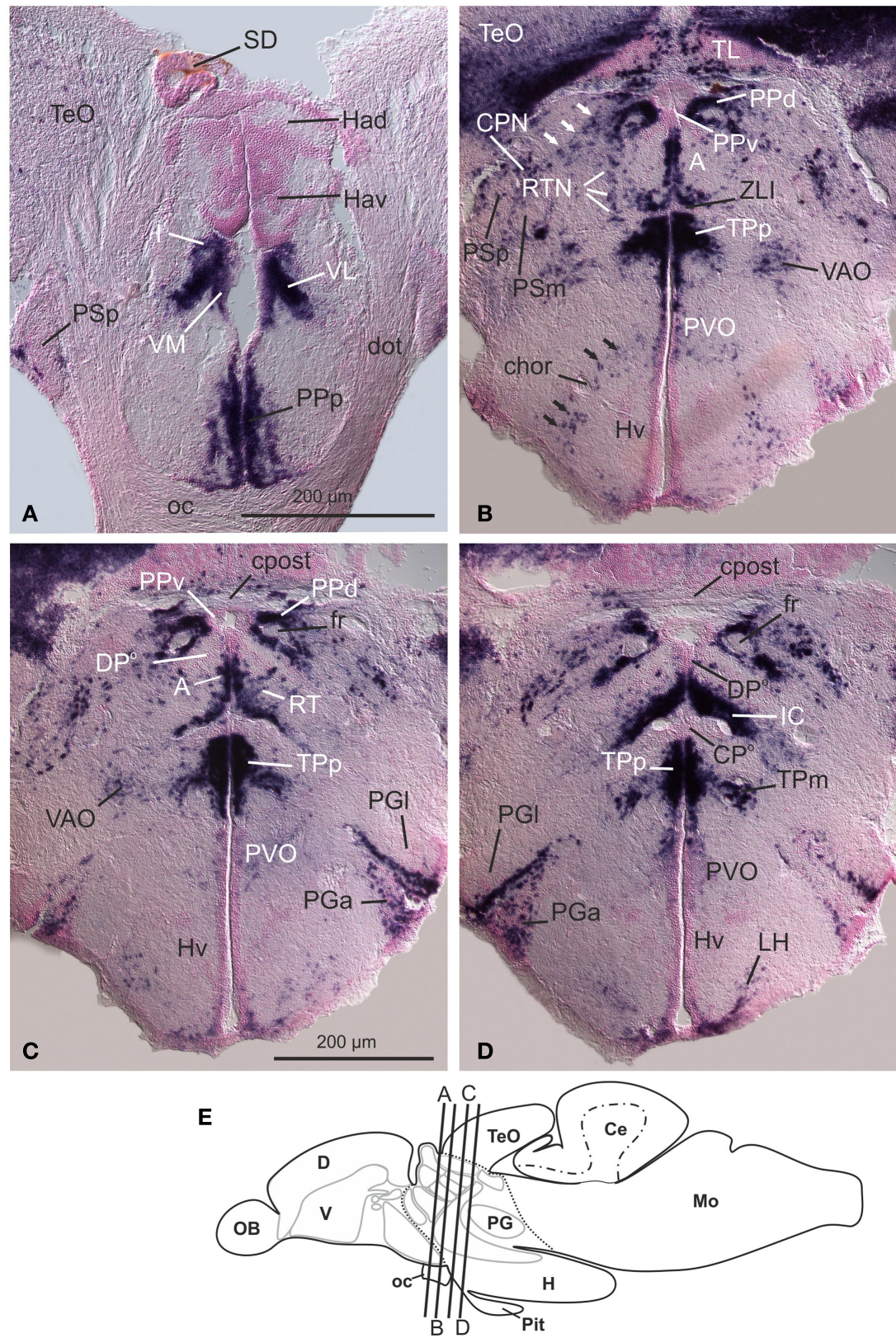

FIGURE 4 | Distribution of GAD67-mRNA reveals prosomerically organized thalamic derivatives in the brain of adult zebrafish (A-D). Ventral thalamic (prethalamic), GABAergic nuclei such as the intermediate, ventrolateral, and ventromedial nuclei $(\mathrm{I}, \mathrm{VL}$, and $\mathrm{VM}$ in $(\mathbf{A})$ are defined by expression of GAD67-mRNA. In the longitudinal view, these nuclei are located anterior to the zona limitans intrathalamica (ZLI, nicely displayed in

(B). Dorsal thalamic nuclei, such as the habenular nuclei [Had, Hav in (A)], and dorsoposterior $\left(\mathrm{DP}^{\circ}\right)$ and centroposterior $\left(\mathrm{CP}^{\circ}\right)$ nuclei, are predominantly glutamatergic and GAD67-negative. However, some dorsal thalamic nuclei close to the $\mathrm{ZLI}$ are also GABAergic and likely derivatives of the rostral thalamus proper (rTh). These are the anterior $(\mathbf{A}, \mathbf{B})$ and the intercalated nucleus [IC; (D)]. (E) Schematic drawing of the zebrafish brain that indicates the approximate position of transverse sections. From Mueller and Guo (2009). Abbreviations see list.
(Dp; Wullimann et al., 1996). In other teleost fish, the EmT is sometimes erroneously considered the proliferative zone of the nucleus taeniae (Nieuwenhuys, 2011).

\section{THE PREGLOMERULAR COMPLEX, A CONVERGENT THALAMUS-LIKE AREA IN TELEOSTS}

After examining thalamic nuclei of the alar plate portions of prosomeres 2 and 3, I now move to those structures usually treated as basal plate derivatives of prosomeres 2 and 3: the periventricular posterior tuberculum and its migrated neighbor, the preglomerular complex. Both are important for the comparative interpretation of the teleostean wider thalamus. This is the case because in teleosts the nuclei of the preglomerular complex in contrast to those of the thalamus proper - are reciprocally connected with pallial parts, mostly the mediodorsally located pallial amygdala (VP/Dm) and the laterodorsal hippocampal 


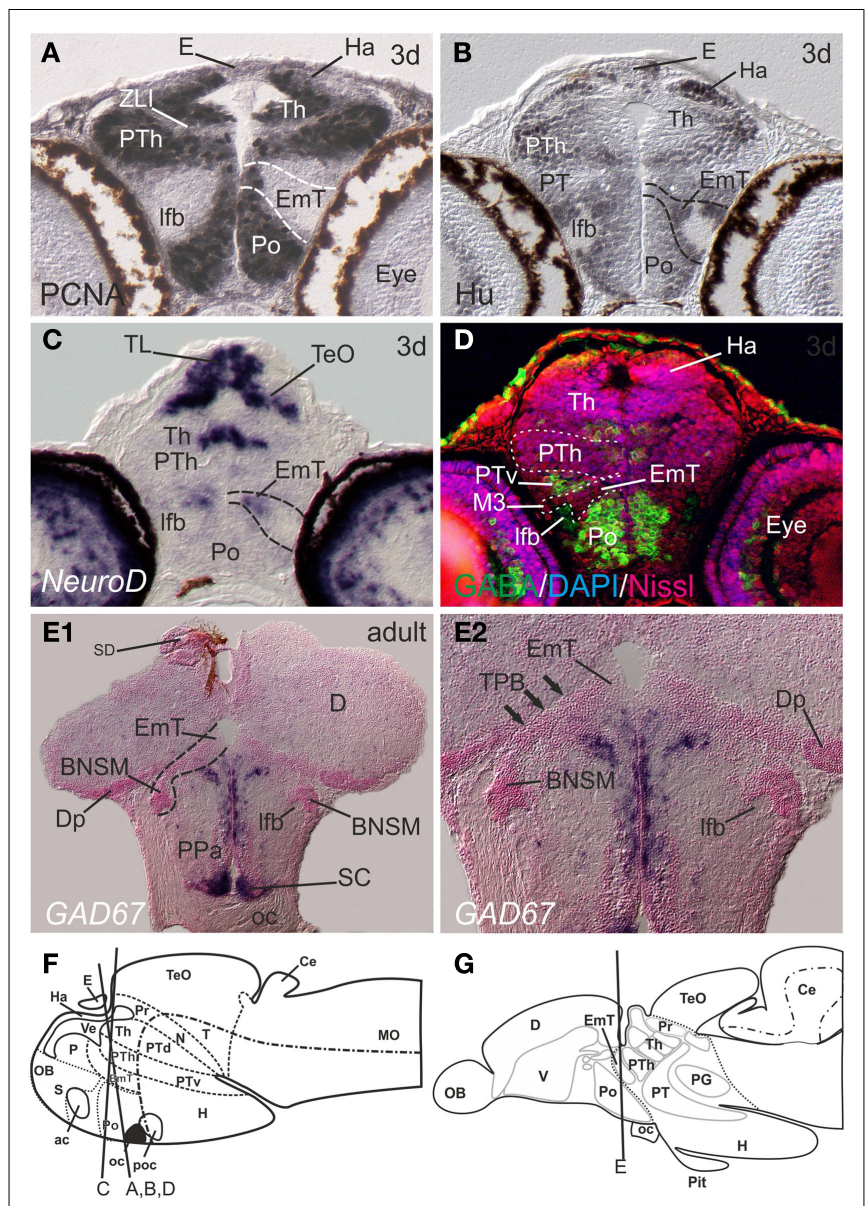

FIGURE 5 |The location of the eminentia thalami (EmT) in postembryonic and adult zebrafish. (A-E) In larval zebrafish, the periventricular proliferative zone [PCNA-positive in (A) and Hu-negative in (B)] of the EmT is located between ventral thalamus (VT) and preoptic region (Po). (C) Postmitotic EmT-cells expressing neuroD-mRNA migrate toward the lateral forebrain bundle (lfb). (D) Both, proliferative and postmitotic cells of the EmT in the larval brain are free of GABA cells, whereas surrounding regions, such as the ventral posterior tuberculum (PTV) and preoptic region (Po) show many GABA cells. Note, the ventral thalamus is usually defined by a heavy presence of GABA-expressing cells: here, the ventral thalamus appears GABA-negative, because its proliferative zone is displayed only. (E1 + E2) The EmT in the brain of adult zebrafish is located ventrally adjacent to the posterior zone $(\mathrm{Dp})$ of the pallium, ventrally to the thalamo-pallial border. The derivative of the EmT, the peripheral bed nucleus of the stria medullaris (BNSM, former ventral nucleus of the entopeduncular complex) surrounds the lateral forebrain bundle. Schematic drawings of larval (F) and adult (G) zebrafish brain that indicates the approximate position of transverse sections. Abbreviations see list.

division $(\mathrm{MP} / \mathrm{Dl})$. Indeed, the majority of the ascending sensory system in teleosts reaches the pallium through the preglomerular complex. It has been suggested, that specific sensory nuclei in the preglomerular complex exist for gustatory, somatosensory, auditory, and visual systems (Wullimann, 1998; Wullimann and Mueller, 2004b). Recent connectivity studies in goldfish, however, show that the majority of these preglomerular nuclei process two or more sensory modalities and, are therefore multimodal (Northcutt, 2006, 2008; Yamamoto and Ito, 2008).
The preglomerular complex in adult cyprinids, like goldfish and zebrafish, consists of a group of satellite nuclei associated with the TGN, which in the past have been termed nucleus glomerulosus (Northcutt, 2008). The preglomerular complex is subdivided in a rostral and a caudal group. The rostral group is formed by three nuclei located at the rostral pole of the TGN: the anterior $(\mathrm{PGa})$, the lateral $(\mathrm{PGl})$, and the medial (PGm) preglomerular nuclei (Figures 6A-D; Figure 6E for overview). The caudal group is formed by the tertiary gustatory nucleus and the closely associated posterior thalamic (P) and subglomerular (SG) nuclei (Figures 6A-D).

In goldfish, the anterior nucleus (PGa) is a predominantly auditory relay nucleus projecting to the dorsomedially positioned pallial amygdala (VP/Dm). It receives projections from the auditory central torus semicircularis and from other auditory nuclei, such as the perilemniscular nucleus, the anterior tuberal nucleus, and the medial pretoral nucleus (Yamamoto and Ito, 2008). The lateral preglomerular nucleus (PGl) receives multiple inputs and is subdivided into different rostral and caudal parts. Different parts of the rostrolateral PGl convey visual, lateral line, and auditory ascending information (goldfish: Yamamoto and Ito, 2008; catfish: Striedter, 1990). The TGN and the closely associated posterior thalamic nuclei receive primarily gustatory projections (crucian carp: Morita et al., 1980, 1983).

Conflicting interpretations exist in regard to possible homologies of nuclei of the preglomerular complex with nuclei of the mammalian thalamus proper. It is assumed that the preglomerular complex evolved in the common ancestor of chondrostean sturgeons and teleosts as a derivative of the posterior tuberculum (Bergquist, 1932; Northcutt, 2008). Based on Pax6 expression patterns in developing medaka, brains, parts if not all of the preglomerular complex are hypothesized as homologous to the thalamus proper (P2) of other vertebrates (Ishikawa et al., 2007). Yet, only sparse data supports this finding. Within the rostral group, for example, the predominantly glutamatergic lateral nucleus of the preglomerular complex (PGl) might indeed share its developmental origin with the centroposterior thalamus $\left(\mathrm{CP}^{\mathrm{O}}\right)$, because in anterior sections the PGI is positioned in the ventrally bent, radial domain of the centroposterior thalamic nucleus (Figure 6A). Both of these nuclei $\left(\mathrm{CP}^{\mathrm{O}}\right.$ and $\mathrm{PGl}$ ) are largely free of GAD67-expression in adult zebrafish. Yet detailed fate mapping studies that could clarify the situation are missing. Some PCNA, BrdU, and neuroD-gene expression studies in brains of zebrafish larvae between 2 and 5 days postfertilization indicate chains of migrating cells from the pretectum, the thalamus proper, and the prethalamus toward the primordial preglomerular complex (M2). All of these proliferation zones may contribute to the preglomerular complex in addition to the cells that derive from the posterior tubercular (bP2/3) proliferation zones (Wullimann and Puelles, 1999; Mueller and Wullimann, 2002).

Outgroup comparisons do also not support a homology between nuclei of the teleostean preglomerular complex and the thalamus proper of other vertebrates. Basal ray-finned fish like cladistian bichirs possess a well-developed posterior tuberculum but lack a preglomerular complex. The same holds true for basal sistergroups of ray-finned fish, such as cartilaginous sharks, manta rays, and agnathan lampreys. According to Northcutt (2006), the preglomerular complex evolved in the last common ancestor of 

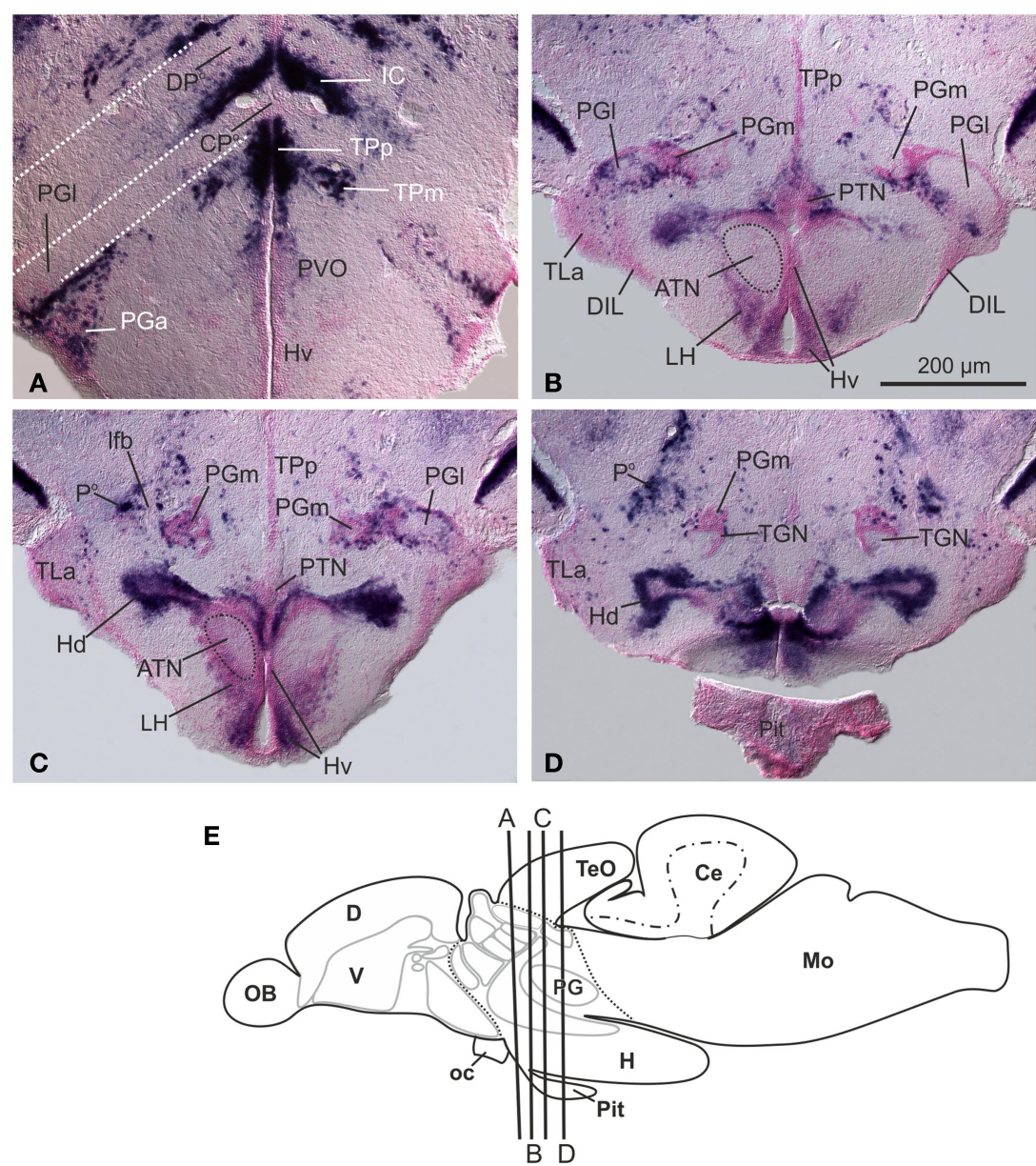

FIGURE 6 | Distribution of GAD67-mRNA at the level of the preglomerular complex and hypothalmus (A-D). Note that the anterior part of the lateral preglomerular nucleus [PGI in (A)] lies in the radial domain of the centroposterior thalamic nucleus [CPo; $(\mathbf{A})$ is a magnification of Figure $\mathbf{4 D}$ ]. The so-called posterior thalamic nucleus [ $P^{\circ}$ in (D)] of the preglomerular complex consists of mostly GAD67-expressing, GABAergic cells. (E) Schematic drawing of the zebrafish brain that indicates the approximate position of transverse sections. From Mueller and Guo (2009). Abbreviations see list. sturgeons and teleosts and is more complex in the latter group. We need then to assume that the teleostean preglomerular complex is a distinctive anatomical feature, an autapomorphy, and a convergent, thalamus-like sensory relay station. In addition, most of the preglomerular complex processes more than one modality which makes its functional organization distinctly different from the mammalian thalamus (Northcutt, 2008).

\section{THE AUDITORY THALAMUS: A CASE OF CONSERVATISM}

The topological organization of thalamic and prethalamic derivatives in teleosts resembles the situation in anamniote tetrapods (amphibians: Neary and Northcutt, 1983; Brox et al., 2003). Their functional significance and connectivity, however, is still poorly understood. Teleost fish do possess a well-described, effective auditory system, which is basically formed by the same central nervous system components found in tetrapod vertebrates (McCormick, $1982,1992)$. This makes the auditory system a perfect candidate to trace how sensory information is relayed through the teleostean thalamus proper in comparison to mammals. In contrast, the visual system in teleosts appears to be very different from the mammalian one displaying widespread retinofugal projections to the preglomerular complex, prethalamic, pretectal nuclei, as well as to the tectum opticum.

Teleosts are capable of performing a range of auditory challenging tasks: they can discriminate distinct frequencies, locate sound sources, and they are capable of analytic and synthetic listening (Schuijf et al., 1977; Fay, 1992; Popper and Fay, 1993). The ears of teleost fish and land vertebrates necessarily differ from each other. Teleosts lack structures directly comparable to either the mammalian cochleae or the amphibian auditory papillae. The inner ear of teleosts processes sound via otolithic endorgans, such as the sacculus, lagena, and the utriculus (Furukawa and Ishii, 1967; Fay, 1978). Sensory fibers innervating the hair cells of the endorgans of the inner ear form the octaval, VIIIth nerve, which transmits both vestibular and acoustic information. The brain area receiving primary auditory information is located in the medulla oblongata and is named after its innervating nerve, the octaval area (Figure 7). This brain region represents the major source of afferents to the auditory torus semicircularis, an alar plate derived midbrain station en route to the teleostean thalamus proper. A column of five nuclei is arranged in a rostrocaudal sequence in the ventral octaval area. These nuclei are the anterior, magnocellular, descending, 


\section{Ascending Auditory Pathway in Rodents}

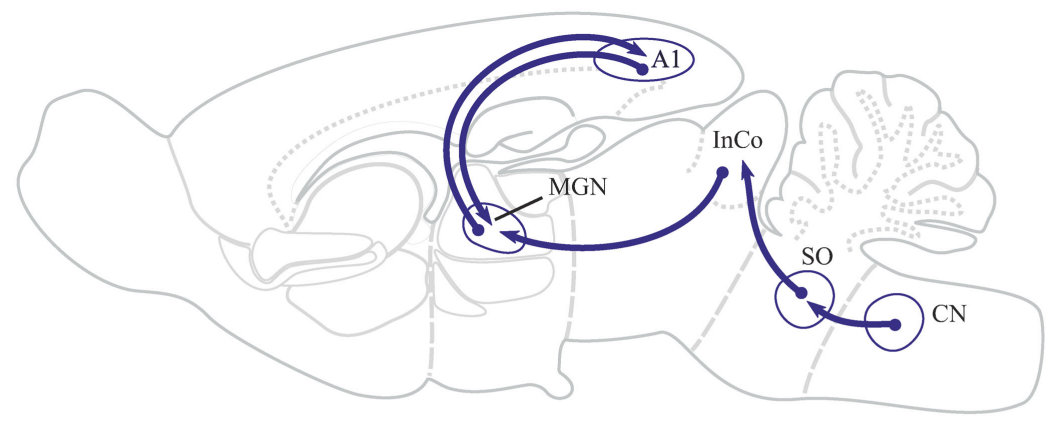

Ascending Auditory Pathway in Cyprinids

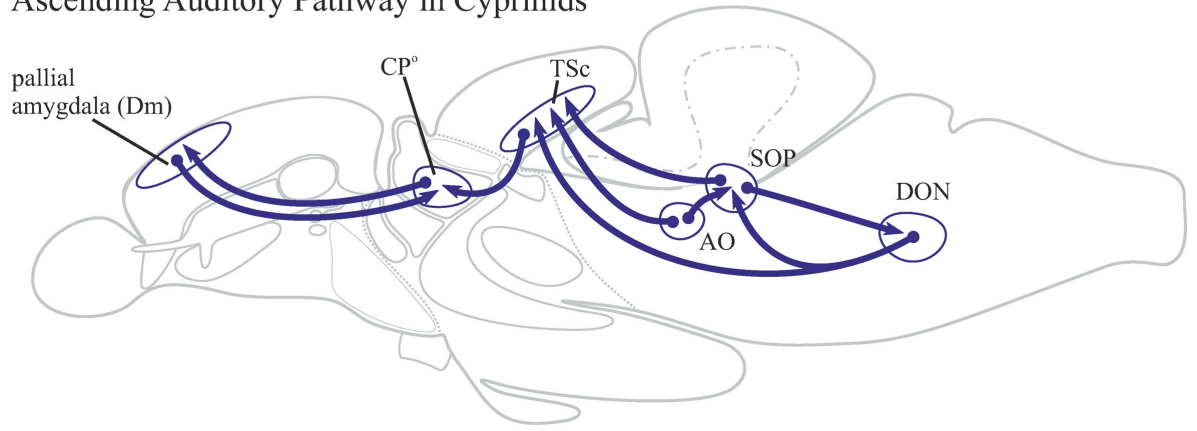

FIGURE 7 | Ascending auditory pathways in rodents and cyprinids. The ascending auditory pathway in cyprinids is similar to those found in mammals. The teleostean secondary octaval population (SOP) is homologous to the mammalian superior olive (SO). The supererior olive (SO) of rodents projects onto the inferior colliculus (IC), which conveys auditory information to the medial geniculate nucleus. The latter is a dorsal thalamic nucleus, which is reciprocally connected with the auditory cortex (A1). In cyprinids, however, the secondary octaval population conveys auditory information to the central nucleus of the torus semicircularis (TSc). This nucleus projects to the auditory centroposterior thalamic nucleus $\left(\mathrm{CP}^{\circ}\right)$, which is reciprocally connected with the dorsomedial located pallial amygdala $(\mathrm{Dm})$ of the telencephalon. The centroposterior nucleus does not project to the dorsal pallium (Dc $=$ central zone) of teleosts. tangential, and posterior nuclei (Northcutt, 1980; McCormick, 1982; Meredith and Butler, 1983).

Primary auditory centers in cyprinids are the dorsal part of the anterior octaval nucleus and the dorsomedial part of the descending octaval nucleus (Echteler, 1984, 1985). Secondary octaval projections from these two nuclei form part of the lateral longitudinal tract in cyprinids and other teleosts which is homologous to the mammalian lateral lemniscus. The name lateral lemniscus comes from the Greek word lemniskos signifying "filet" which refers to the lateral filet-like position of its lemniscal fibers. In teleosts, they project to a medially positioned central nucleus of the torus semicircularis, an auditory nucleus of the dorsal midbrain homologous to the mammalian inferior colliculus (InCo; Figure 7). Another part of the lateral lemniscus in cyprinids projects to a secondary octaval population of neurons, which may be homologous to the superior olive of mammals (Echteler, 1984; McCormick and Hernandez, 1996). The torus semicircularis is reciprocally connected to the auditory centroposterior $\left(\mathrm{CP}^{\mathrm{o}}\right)$ thalamic nucleus in cyprinids and other teleosts. In teleosts, this auditory relay nucleus projects to the dorsomedial lying pallial amygdala (VP/Dm). Thalamocortical projections are absent in teleost fish. The majority of the thalamotelencephalic projections are targeted to the subpallial basal ganglia, the dorsal part (Vd) of the ventral telencephalon (Northcutt, 2006). Currently, the auditory centroposterior $\left(\mathrm{CP}^{\mathrm{o}}\right)$ thalamic nucleus is the only known nucleus reciprocally connected to the pallial amygdala $(\mathrm{VP} / \mathrm{Dm})$ in the cyprinid goldfish and in two vocal batrachoid teleosts, the plainfin midshipman and the gulf toadfish (Goodson and Bass, 2002; Northcutt, 2006).

\section{VISUAL THALAMIC AREAS: A LOST QUEST FOR THE GENICULATE PATHWAY}

The visual system is highly developed in most teleosts. Goldfish and zebrafish are tetrachromats and their color vision is based on four cone types. Mammals, in contrast, are predominantly trichromats possessing three cone types (Neumeyer and Arnold, 1989; Robinson et al., 1993). The retinal ganglion cells of teleosts project to five primary target sites: the optic tectum, the thalamus, the pretectum, the accessory optic system, and the preoptic area (Northcutt and Wullimann, 1988). Two of them will be discussed here: the optic tectum and the thalamic projection sites. The optic tectum is the dominant visual center in the teleostean brain. It processes the vast majority of visual information that concern movement, shape and color of objects. The teleostean optic tectum is homologous to the mammalian superior colliculus, which is a relatively small portion in the dorsal midbrain.

Studies that describe visual thalamic circuits in teleosts (Figure 8) and other ray-finned fish in comparison to the mammalian situation focus on geniculate and extrageniculate visual pathways (Wullimann, 1998). In mammals around $90 \%$ of the retinal fibers ascend to the dLGN. This thalamic sensory nucleus 


\section{Ascending Visual Pathways in Rodents}
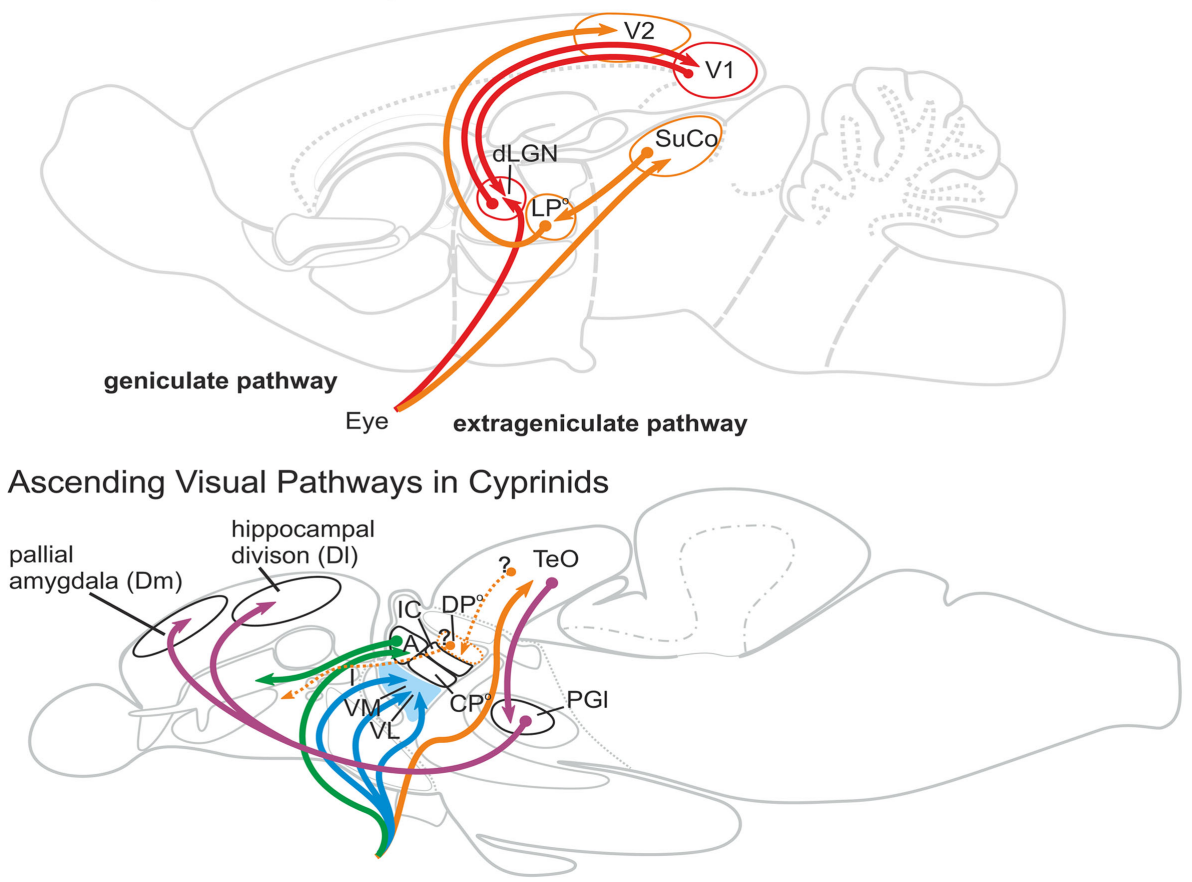

FIGURE 8 | Ascending visual pathways in rodents and cyprinids. In mammals, $90 \%$ of the primary visual projections target the dorsal lateral geniculate nucleus (dLGN), which is reciprocally connected with the primary visual cortex (V1). This pathway is called the geniculate pathway (red). The extrageniculate pathway (orange) comprises primary visual projections that target the superior colliculus (SuCo). The superior colliculus projects to the lateroposterior dorsal thalamic complex $\left(\mathrm{LP}^{\circ}\right)$ of rodents. The lateroposterior complex is reciprocally connected with the secondary visual cortex (V2). Ascending visual pathways in goldfish could not establish geniculate and extrageniculate pathways. Primary visual projections target, for example, ventral thalamic nuclei (blue: intermediate (I), ventromedial (VM), ventralateral
$(V L)$ thalamic nuclei), as well as the anterior nucleus (green, A), and the tectum opticum (orange) which is homologous to the mammalian superior colliculus (SuCo). Based on studies in some teleosts, the dorsoposterior thalamic nucleus ( $\mathrm{DP}^{\circ}$ ) of cyprinids does likely receive visual information from the tectum opticum ( $\mathrm{TeO}$ ) and, thus, likely represents the visual thalamic nucleus. However, this nucleus does project to the striatopallidum (Vd), but not to any pallial area. The only thalamus-like visual relay nucleus is the lateral preglomerular nucleus, which projects to the dorsomedial pallial amygdala $(\mathrm{Dm})$ and to the laterodorsally located hippocampal divsion (DI; projections in purple). Thus, geniculate and extrageniculate pathways as found in mammals are absent in teleosts. relays primary visual information to $\mathrm{V} 1$, an important entrance part of the visual cortex. This visual pathway is called the geniculate pathway. In contrast, the mammalian extrageniculate pathway is characterized by retinal projections to the superior colliculus, which projects to a group of caudal thalamic nuclei, mainly the pulvinar or lateroposterior complex. These tectorecipient thalamic visual nuclei project to all visual cortical areas (V2-V4) except to V1 and to the lateral amygdala (Doron and Ledoux, 1999).

During the eighties when tracers such as horseradish peroxidase and DiI became available, retinothalamic projections were investigated in a variety of teleost fishes, particularly in goldfish. Northcutt and Wullimann (1988) reviewed these visual tracing studies for the revised nomenclature of the teleostean diencephalon provided by Braford and Northcutt (1983). Ascending visual pathways are schematically summarized in Figure 8. Thalamic projection sites found in all teleosts include primarily prethalamic derivatives: the ventromedial (VM), intermediate (I), and ventrolateral (VL) nuclei. Connectivity and comparability to amniote vertebrates regarding these prethalamic nuclei are unknown (Butler and Hodos, 2005). The anterior thalamic nucleus (A) is the only teleostean thalamic nucleus, which receives primary visual projections and projects to a part of the telencephalon, the region that is interpreted as the striatopallidum $(\mathrm{Vd})$. As a retinorecipent thalamic nucleus, the anterior thalamic nucleus (A) has either been discussed as a field homolog of the dorsal geniculate nucleus (Butler and Hodos, 2005) or it has been considered part of the geniculate pathway (Wullimann, 1998). Yet like its amphibian homolog, the teleostean anterior thalamic nucleus is a GABAergic dorsal thalamic nucleus and likely a derivative of the newly defined rostral thalamus proper. In contrast, the mammalian dLGN is glutamatergic and probably a derivative of the cTh proper. A teleostean nucleus homologous to the mammalian dLGN might be absent in teleosts, similar to what has been found in amphibians (Roth et al., 2003).

Do teleosts possess a tectorecipient dorsal thalamic nucleus that serves as an extrageniculate pathway to the telencephalon? The mammalian pulvinar/lateroposterior complex is a dorsal thalamic structure that receives visual projections from the superior colliculus. Nuclei of this structure predominantly project to all primary visual cortical areas outside V1. Echteler and Saidel (1981) suggested that the dorsoposterior thalamic nucleus $\left(\mathrm{DP}^{\mathrm{o}}\right)$ in goldfish serves as a visual thalamic relay nucleus which projects to the 
dorsomedial (Dm) part of the pallium. Recent DiI tracing studies in goldfish, though, demonstrate that the dorsal posterior nucleus in goldfish projects to the subpallium and not to pallial domains such as the putative homologs of the amygdala, the hippocampus, or the cortex (Northcutt, 2006). Tracing studies in the elephantnose fish (Gnathonemus petersii) show projections from the optic tectum to the dorsal posterior thalamic nucleus supporting a visual function. Also a homologous nucleus in the teleost catfish (Ictalurus punctatus) receives tectal projections (Striedter, 1990). Corresponding findings are still lacking for cyprinids like carp, goldfish, or zebrafish. Two reports in goldfish did not demonstrate projections from the tectum to the dorsoposterior nucleus (Sharma, 1975; Luiten, 1981).

Taken together, in cyprinids thalamic regions involved in visual processing clearly differ from mammalian visual systems (Figure 8). The teleostean ascending visual system can hardly be compared to the mammalian extrageniculate pathway. There is currently no evidence for a nucleus of the thalamus proper projecting to any other part of the pallium like the pallial amygdala, the dorsal pallium, or the hippocampal division. Thus, teleosts do not have homologous visual thalamoamygdalar projections. Future research needs to clarify if the dorsal posterior thalamic nucleus in cyprinids receives tectal input and if is functions are restricted to visual processing only.

\section{CONCLUSION}

The thalamus proper of placental mammals is a complex structure in the caudal diencephalon. It serves as a sensory relay station connecting the isocortex with the larger brain. What is the thalamus in zebrafish? Conserved patterning mechanisms and

\section{REFERENCES}

Amo, R., Aizawa, H., Takahoko, M., Kobayashi, M., Takahashi, R., Aoki, T., and Okamoto, H. (2010). Identification of the zebrafish ventral habenula as a homolog of the mammalian lateral habenula. J. Neurosci. 30, 1566-1574.

Bergquist, H. (1932). Zur Morphologie des Zwischenhirns bei niederen Wirbeltieren. Acta Zool. 13, 57-304.

Braford, M., and Northcutt, R. (1983). "Organization of the diencephalon and pretectum of the ray-finned fishes," in Fish Neurobiology, eds D. Re and N. Rg (Ann Arbor, University of Michigan Press), 117-140.

Brox, A., Puelles, L., Ferreiro, B., and Medina, L. (2003). Expression of the genes GAD67 and Distal-less4 in the forebrain of Xenopus laevis confirms a common pattern in tetrapods. J. Comp. Neurol. 461, 370-393.

Butler, A. B. (1995). The dorsal thalamus of jawed vertebrates: a comparative viewpoint. Brain Behav. Evol. 46, 209-223.

Butler, A. B., and Hodos, W. (2005). Comparative Vertebrate tation. Hoboken, NJ: John Wiley and Sons.

Butler, A. B., and Northcutt, R. G. (1993). The diencephalon of the Pacific herring, Clupea harengus: cytoarchitectonic analysis. J. Comp. Neurol. 328, 527-546.

Doron, N. N., and Ledoux, J. E. (1999). Organization of projections to the lateral amygdala from audimus in the rat. J. Comp. Neurol. 412, 383-409.

Echteler, S. M. (1984). Connections of the auditory midbrain in a teleost fish, Cyprinus carpio. J. Comp. Neurol. 230, 536-551.

Echteler, S. M. (1985). Tonotopic organization in the midbrain of a teleost fish. Brain Res. 338, 387-391.

Echteler, S. M., and Saidel, W. M. (1981). fish support telencephalic homologies with land vertebrates. Science 212, 683-685.

Fay, R. R. (1978). Coding of information in single auditory-nerve fibers of the goldfish. J. Acoust. Soc. Am. 63, 136-146.
Neuroanatomy: Evolution and Adaptory and visual areas of the thalaForebrain connections in the gold-

similar processes during neurogenesis allow for a comparison between zebrafish and mammals at early stages of development. The thalamus proper in adult zebrafish, however, strongly differs from the mammalian one. The question "What is the Zebrafish Thalamus?" then is truly a question after (1) the elements in the zebrafish brain that correspond topologically and (2) the elements that execute similar connectivity and functions. Certain nuclei of the thalamus proper, such as the anterior (A) and the dorsoposterior $\left(\mathrm{DP}^{\mathrm{o}}\right)$ thalamic nuclei apparently correspond topologically to derivatives of the rostral and caudal Thalamus respectively. Unlike counterparts in mammals, their projections however are targeted to the ventral telencephalon and not to a region that corresponds to the mammalian isocortex. Defining features of the mammalian thalamus proper, such as thalamocortical connections and the dLGN, are absent in zebrafish. Most importantly, it is the preglomerular complex and not the thalamus proper that act in zebrafish as major sensory relay station. Zebrafish as a genetically traceable model organism has only begun to reveal the developmental processes and neural circuits defining the thalamus in teleosts. Genetic approaches that include molecular-anatomical analyses of late developing and mature zebrafish brains are needed to solve the evolutionary questions outlined here.

\section{ACKNOWLEDGMENTS}

I thank Wolfgang Driever and Alida Filippi for critical comments on the manuscript, and Steffi Dippold for her editorial work. This work was supported by research grants to Wolfgang Driever from EU FP7 (ZF-HEALTH/Proj. No. 242048) and the Freiburg Institute for Advanced Studies.

Fay, R. R. (1992). Analytic listening by the goldfish. Hear. Res. 59, 101-107.

Furukawa, T., and Ishii, Y. (1967). Neurophysiological studies on hearing in goldfish. J. Neurophysiol. 30, 1377-1403.

Goodson, J. L., and Bass, A. H (2002). Vocal-acoustic circuitry and descending vocal pathways in teleost fish: convergence with terrestrial vertebrates reveals conserved traits. J. Comp. Neurol. 448 , 298-322.

Hennig, W. (1966). Phylogenetic Systematics. Urbana: University of Illinois Press.

Inamura, N., Ono, K., Takebayashi, H., Zalc, B., and Ikenaka, K. (2011). Olig2 lineage cells generate GABAergic neurons in the prethalamic nuclei, including the zona incerta, ventral lateral geniculate nucleus and reticular thalamic nucleus. Dev. Neurosci. 33, 118-129.

Ishikawa, Y., Yamamoto, N., Yoshimoto, M., Yasuda, T., Maruyama, K., Kage, T., Takeda, H., and Ito, H. (2007). Developmental origin of diencephalic sensory relay nuclei in teleosts. Brain Behav. Evol. 69, 87-95.
Jones, E. G. (2007). The Thalamus. Cambridge: Cambridge University Press.

Jones, E. G., and Rubenstein, J. L. (2004). Expression of regulatory genes during differentiation of thalamic nuclei in mouse and monkey. J. Comp. Neurol. 477, 55-80.

Kaas, J. H. (2009). Evolutionary Neuroscience. Oxford: Academic Press.

Kataoka, A., and Shimogori, T. (2008). Fgf8 controls regional identity in the developing thalamus. Development 135, 2873-2881.

Loopuijt, L. D., Villablanca, J. R., and Hovda, D. A. (1995). Morphological changes in the thalamus and neocortex of the cat brain after a restricted unilateral fetal neocortical lesion. Brain Res. Dev. Brain Res. 85, 259-272.

Luiten, P. G. (1981). Afferent and efferent connections of the optic tectum in the carp (Cyprinus carpio L.). Brain Res. 220, 51-65.

McCormick, C. A. (1982). The organization of the octavolateralis area in actinopterygian fishes: a new interpretation. J. Morphol. 171, 159-181. 
McCormick, C. A. (1992). "Evolution of central auditory pathways in anamniotes," in The Evolutionary Biology of Hearing, eds R. R. Webster and A. N. Popper (New York: Springer), 323-350.

McCormick, C. A., and Hernandez, D. V. (1996). Connections of octaval and lateral line nuclei of the medulla in the goldfish, including the cytoarchitecture of the secondary octaval population in goldfish and catfish. Brain Behav. Evol. 47, 113-137.

Meredith, G. E., and Butler, A. B. (1983). Organization of eighth nerve afferent projections from individual endorgans of the inner ear in the teleost, Astronotus ocellatus. J. Comp. Neurol. 220, 44-62.

Meek, J., and Nieuwenhuys, R. (1998). "Holosteans and teleosts," in The Central Nervous System of Vertebrates, eds R. Nieuwenhuys, H. J. Ten Donkelaar, and C. Nicholson (Berlin: Springer), 760-937.

Morita, Y., Ito, H., and Masai, $\mathrm{H}$. (1980). Central gustatory paths in the crucian carp, Carassius carassius. J. Comp. Neurol. 191, 119-132.

Morita, Y., Murakami, T., and Ito, H. (1983). Cytoarchitecture and topographic projections of the gustatory centers in a teleost, Carassius carassius. J. Comp. Neurol. 218, 378-394.

Mueller, T., Dong, Z., Berberoglu, M. A., and Guo, S. (2011). The dorsal pallium in zebrafish, Danio rerio (Cyprinidae, Teleostei). Brain Res. 1381, 95-105.

Mueller, T., and Guo, S. (2009). The distribution of GAD67-mRNA in the adult zebrafish (teleost) forebrain reveals a prosomeric pattern and suggests previously unidentified homologies to tetrapods. J. Comp. Neurol. 516, 553-568.

Mueller, T., Vernier, P., and Wullimann, M. F. (2006). A phylotypic stage in vertebrate brain development: GABA cell patterns in zebrafish compared with mouse. J. Comp. Neurol. 494, 620-634.

Mueller, T., and Wullimann, M. F. (2002). BrdU-, neuroD (nrd)- and Hu-studies reveal unusual nonventricular neurogenesis in the postembryonic zebrafish forebrain. Mech. Dev. 117, 123-135.

Mueller, T., and Wullimann, M. F. (2009). An evolutionary interpretation of teleostean forebrain anatomy. Brain Behav. Evol. 74, 30-42.

Nakagawa, Y., and O'Leary, D. D. (2001). Combinatorial expression patterns of LIM-homeodomain and other regulatory genes parcellate developing thalamus. J. Neurosci. 21, 2711-2725.
Neary, T. J., and Northcutt, R. G. (1983). Nuclear organization of the bullfrog diencephalon. J. Comp. Neurol. 213, 262-278.

Neumeyer, C., and Arnold, K. (1989). Tetrachromatic color vision in the goldfish becomes trichromatic under white adaptation light of moderate intensity. Vision Res. 29, 1719-1727.

Nieuwenhuys, R. (1963). The comparative anatomy of the actinopterygian forebrain. J. Hirnforsch. 171-192.

Nieuwenhuys, R. (2011). The development and general morphology of the telencephalon of actinopterygian fishes: synopsis, documentation and commentary. Brain Struct. Funct. 215, 141-157.

Nieuwenhuys, R., Ten Donkelaar, H. J., and Nicholson, C. (1998). The Central Nervous System of Vertebrates. Berlin: Springer.

Nieuwenhuys, R., and Meek, J. (1990). "The telencephalon of actinopterygian fishes," in Cerebral Cortex, Vol. 8A, eds E. G. Jones and A. Peters (New York: Plenum Press), 31-73.

Nieuwenhuys, R., Voogd, J., and Van Huijzen, C. (2007). The Human Central Nervous System. Berlin: Springer.

Northcutt, R. G. (2006). Connections of the lateral and medial divisions of the goldfish telencephalic pallium. J. Comp. Neurol. 494, 903-943.

Northcutt, R. G. (2008). Forebrain evolution in bony fishes. Brain Res. Bull. 75, 191-205.

Northcutt, R. G. (ed.). (1980). Central Auditory Pathways in Anamniote Vertebrates. New York: Springer-Verlag.

Northcutt, R. G., and Davis, R. E. (1983). "Telencephalic organization in ray-finned fishes," in Higher Brain Areas and Functions, eds R. E. Davis and R. G. Northcutt (Ann Arbor: The University of Michigan Press), 203-236.

Northcutt, R. G., and Wullimann, M. F. (1988). "The visual system in teleost fishes: morphological patterns and trends," in Sensory Biology of Aquatic Animals, eds J. Atema, R. R. Fay, A. N. Popper, and W. N. Tavolga (New York, Springer), 515-552.

Osório, J., Mueller, T., Retaux, S., Vernier, P., and Wullimann, M. F. (2010). Phylotypic expression of the bHLH genes Neurogenin2, Neurod, and Mash1 in the mouse embryonic forebrain. J. Comp. Neurol. 518, 851-871.

Peukert, D., Weber, S., Lumsden, A., and Scholpp, S. (2011). Lhx2 and Lhx 9 determine neuronal differentiation and compartition in the caudal forebrain by regulating wnt signaling. PLoS Biol. 9, e1001218. doi:10.1371/journal.pbio.1001218

Popper, A. N., and Fay, R. R. (1993). Sound detection and processing by fish: critical review and major research questions. Brain Behav. Evol. 41, 14-38.

Portavella, M., Vargas, J. P., Torres, B., and Salas, C. (2002). The effects of telencephalic pallial lesions on spatial, temporal, and emotional learning in goldfish. Brain Res. Bull. 57, 397-399.

Puelles, L., and Rubenstein, J. L. (1993). Expression patterns of homeobox and other putative regulatory genes in the embryonic mouse forebrain suggest a neuromeric organization. Trends Neurosci. 16, 472-479.

Puelles, L., and Rubenstein, J. L. (2003). Forebrain gene expression domains and the evolving prosomeric model. Trends Neurosci. 26, 469-476.

Rink, E., and Wullimann, M. F. (2002). Connections of the ventral telencephalon and tyrosine hydroxylase distribution in the zebrafish brain (Danio rerio) lead to identification of an ascending dopaminergic system in a teleost. Brain Res. Bull. 57, 385-387.

Rink, E., and Wullimann, M. F. (2004). Connections of the ventral telencephalon (subpallium) in the zebrafish (Danio rerio). Brain Res. 1011, 206-220

Robertson, B., Auclair, F., Menard, A., Grillner, S., and Dubuc, R. (2007). GABA distribution in lamprey is phylogenetically conserved. J. Comp. Neurol. 503, 47-63.

Robinson, J., Schmitt, E. A., Harosi, F. I., Reece, R. J., and Dowling, J. E. (1993). Zebrafish ultraviolet visual pigment: absorption spectrum, sequence, and localization. Proc. Natl. Acad. Sci. U.S.A. 90, 6009-6012.

Rose, J. E., and Woolsey, C. N. (1943). A study of thalamo-cortical relations in the rabbit. Bull. Johns Hopkins Hosp. 123, 65-123.

Roth, G., Grunwald, W., and Dicke, U. (2003). Morphology, axonal projection pattern, and responses to optic nerve stimulation of thalamic neurons in the fire-bellied toad Bombina orientalis. J. Comp. Neurol. 461, 91-110.

Rupp, B., and Northcutt, R. G. (1998). The diencephalon and pretectum of the white sturgeon (Acipenser transmontanus): a cytoarchitectonic study. Brain Behav. Evol. 51, 239-262.

Rupp, B., Wullimann, M. F., and Reichert, H. (1996). The zebrafish brain: a neuroanatomical comparison with the goldfish. Anat. Embryol. 194, 187-203.

Ryu, S., Holzschuh, J., Mahler, J., and Driever, W. (2006). Genetic analysis of dopaminergic system development in zebrafish. J. Neural Transm. Suppl. 61-66.

Ryu, S., Mahler, J., Acampora, D., Holzschuh, J., Erhardt, S., Omodei, D., Simeone, A., and Driever, W. (2007). Orthopedia homeodomain protein is essential for diencephalic dopaminergic neuron development. Curr. Biol. 17, 873-880.

Scholpp, S., Delogu, A., Gilthorpe, J., Peukert, D., Schindler, S., and Lumsden, A. (2009). Her6 regulates the neurogenetic gradient and neuronal identity in the thalamus. Proc. Natl. Acad. Sci. U.S.A. 106, 19895-19900.

Scholpp, S., and Lumsden, A. (2010). Building a bridal chamber: development of the thalamus. Trends Neurosci. 33, 373-380.

Schuijf, A., Visser, C., Willers, A. F., and Buwalda, R. J. (1977). Acoustic localization in an ostariophysian fish. Experientia 33, 1062-1063.

Sharma, S. C. (1975). Visual projection in surgically created "compound" tectum in adult goldfish. Brain Res. 93, 497-501.

Striedter, G. F. (1990). The diencephalon of the channel catfish, Ictalurus punctatus. II. Retinal, tectal, cerebellar and telencephalic connections. Brain Behav. Evol. 36, 355-377.

Tay, T. L., Ronneberger, O., Ryu, S., Nitschke, R., and Driever, W. (2011). Comprehensive catecholaminergic projectome analysis reveals singleneuron integration of zebrafish ascending and descending dopaminergic systems. Nat. Commun. 2, 171.

Vue, T. Y., Aaker, J., Taniguchi, A., Kazemzadeh, C., Skidmore, J. M., Martin, D. M., Martin, J. F., Treier M., and Nakagawa, Y. (2007). Characterization of progenitor domains in the developing mouse thalamus. J. Comp. Neurol. 505, 73-91.

Vue, T. Y., Bluske, K., Alishahi, A., Yang, L. L., Koyano-Nakagawa, N., Novitch, B., and Nakagawa, Y. (2009). Sonic hedgehog signaling controls thalamic progenitor identity and nuclei specification in mice. J. Neurosci. 29, 4484-4497.

Wullimann, M. F. (1998) "The central nervous system," in The Physiology of Fishes, 2nd Edn, ed. D. H. Evans (Boca Raton, NY: CRC Press), 245-282.

Wullimann, M. F., and Mueller, T. (2004a). Identification and morphogenesis of the eminentia thalami in the zebrafish. J. Comp. Neurol. 471, $37-48$. 
Wullimann, M. F., and Mueller, T. (2004b). Teleostean and mammalian forebrains contrasted: evidence from genes to behavior. J. Comp. Neurol. 475, 143-162.

Wullimann, M. F., and Northcutt, R. G. (1990). Visual and electrosensory circuits of the diencephalon in mormyrids: an evolutionary perspective. J. Comp. Neurol. 297, 537-552.

Wullimann, M. F., and Puelles, L. (1999). Postembryonic neural proliferation in the zebrafish forebrain and its relationship to prosomeric domains. Anat. Embryol. 199, 329-348.

Wullimann, M. F., and Rink, E. (2001). Detailed immunohistology of Pax6 protein and tyrosine hydroxylase in the early zebrafish brain suggests role of Pax6 gene in development of dopaminergic diencephalic neurons. Brain Res. Dev. Brain Res. 131, 173-191.

Wullimann, M. F., Rupp, B., and Reichert, H. (1996). Neuroanatomy of the Zebrafish Brain - A Topological Atlas. Basel: Birkhäuser Verlag.

Yamamoto, N., and Ito, H. (2008). Visual, lateralline, and auditory ascending pathways to the dorsal telencephalic area through the rostrolateral region of the lateral preglomerular nucleus in cyprinids. $J$. Comp. Neurol. 508, 615-647.

Conflict of Interest Statement: The author declares that the research was conducted in the absence of any commercial or financial relationships that could be construed as a potential conflict of interest.

Received: 01 February 2012; paper pending published: 13 February 2012; accepted: 11 April 2012; published online: 07 May 2012.

Citation: Mueller T (2012) What is the thalamus in zebrafish? Front. Neurosci. 6:64. doi: 10.3389/fnins.2012.00064

This article was submitted to Frontiers in Neurogenesis, a specialty of Frontiers in Neuroscience.

Copyright (c) 2012 Mueller. This is an open-access article distributed under the terms of the Creative Commons Attribution Non Commercial License, which permits non-commercial use, distribution, and reproduction in other forums, provided the original authors and source are credited. 